\title{
Modern STEM EBIC: Emerging Modes and Methods
}

\author{
William Hubbard ${ }^{1}$, Matthew Mecklenburg ${ }^{2}$, Jared Lodico ${ }^{3}$, Brian Zutter ${ }^{4}$, Ho Leung Chan $^{4}$ and B. \\ C. $\operatorname{Regan}^{3}$
}

${ }^{1}$ NanoElectronic Imaging, Inc., Los Angeles, California, United States, ${ }^{2}$ Core Center of Excellence in Nano Imaging (CNI), University of Southern California, Los Angeles, California 90089, United States, United States, ${ }^{3}$ Department of Physics and Astronomy, University of California, Los Angeles, California 90095, United States, United States, ${ }^{4}$ University of California, Los Angeles, Los Angeles, California, United States

In use since the 1960's [1], electron beam-induced current (EBIC) imaging is a common tool for mapping electric fields in a scanning electron microscope (SEM). In the "standard" EBIC mode, current is generated through electron-hole pair separation in local electric fields. This standard mode of EBIC can also be performed in a transmission electron microscope in scanning mode (STEM). Recently a new mode of EBIC was demonstrated in STEM, SEEBIC, which measures current generated by the emission of secondary electrons from the sample [2]. These EBIC modes produce completely distinct contrast, which corresponds to entirely different electrical, thermal, and physical features. SEEBIC is also capable of much higher resolution $(\leq 2 \AA)$ than standard EBIC [3]. Here we discuss a variety of new STEM EBIC-based methods for mapping sample properties that are not accessible with standard TEM imaging, including resistance, temperature, and thickness.

The Pt electrodes in Fig. 1A are patterned on a silicon nitride membrane and poor liftoff has left metallic debris between them. SEEBIC images acquired independently from each electrode are used to compute the resistance to each (virtually grounded) electrode [2,4], shown here in a 3D "topographic" representation. The "altitude" (resistance to ground) is lowest on the electrodes, higher in the region with debris, and highest on the clean silicon nitride. We are unaware of any other TEM-based techniques capable of generating contrast related to conductivity.

The carbon electrode in Fig. 1B sits adjacent to a Pt heater. Al nanoparticles, which are used as thermometers for temperature calibration via plasmon energy expansion thermometry (PEET) [5], are drop-cast onto the entire sample. Increasing the heater temperature increases the carbon's SEEBIC signal significantly (Fig. 1B lower image). Similar experiments (not shown) show that standard EBIC has a similar, but much stronger, temperature response. Temperature mapping with sensitivity of just a few Kelvin is possible in both EBIC modes. Temperature mapping (i.e. with thousands of pixels) in the TEM has otherwise only been demonstrated with PEET, and all other known methods only work for a narrow range of materials. In contrast, a broad range of materials is subject to STEM EBIC thermometry: In Fig. 1B similar temperature measurements are acquired on the amorphous carbon, on crystalline $\mathrm{Al}$ nanoparticles, and on the insulating, amorphous silicon nitride.

Unlike in Figs. 1A and 1B, where the EBIC electrode is in the field of view, in the Fig. 1C images of a (roughly spherical) tin nanoparticle the EBIC is measured remotely and is found to provide a precise measure of thickness. The ADF STEM line profile (Fig. 1C plot) shows that the thick center of the nanoparticle attenuates the number of electrons scattering at high angles ( $>60 \mathrm{mrad}$ ) into the detector. The thickness map, calculated from the remotely acquired EBIC signal, shows its strongest signal in the middle of the nanoparticle and a thickness profile consistent with a sphere of homogeneous density. 
Preliminary results suggest that this technique is capable of thickness resolution comparable to AFM height mapping with lateral resolution comparable to standard STEM imaging.
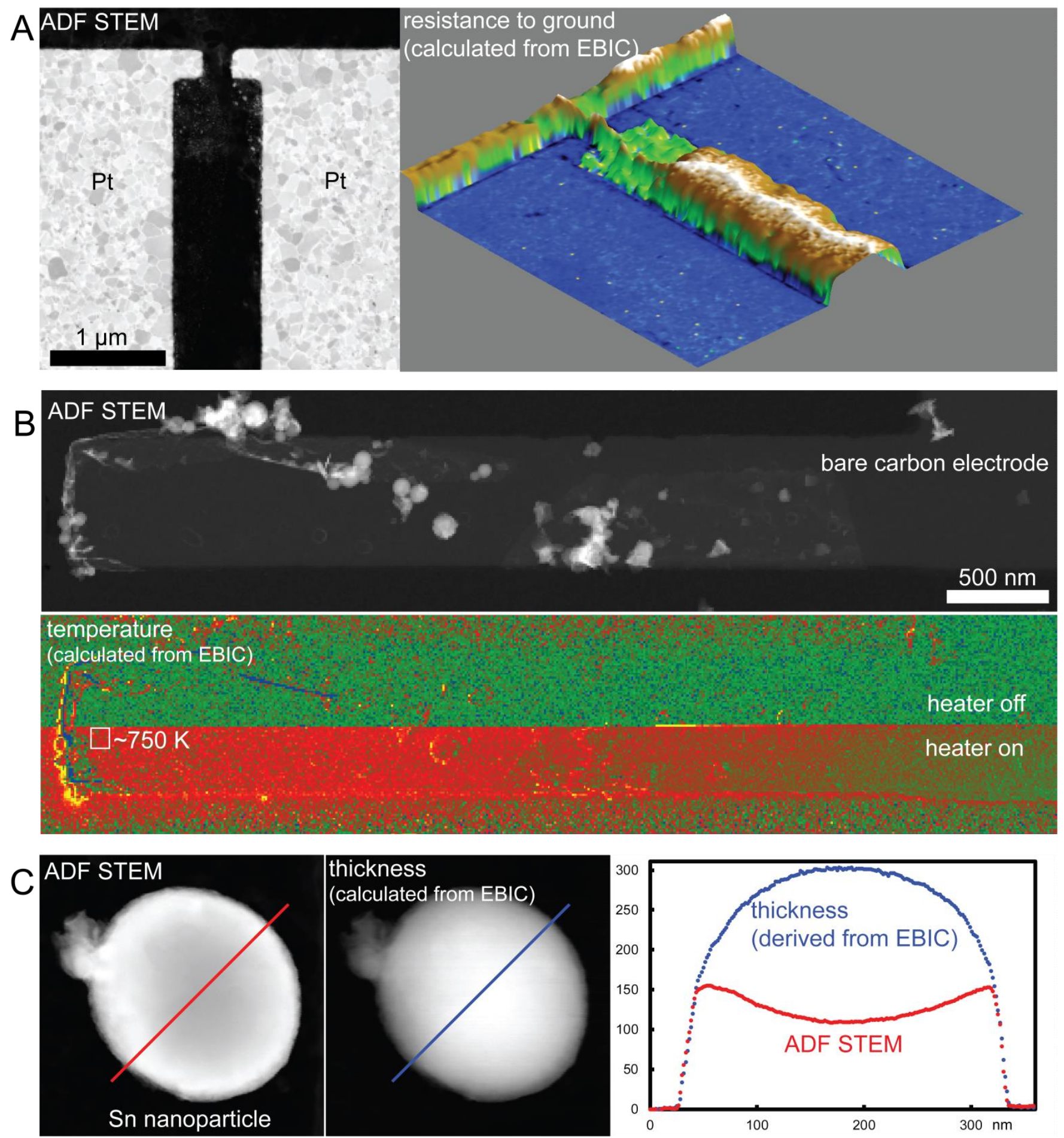

References

[1] TE Everhart, OC Wells, and RK Matta, Proceedings of the IEEE, 52 (1964). p. 1642-1647

[2] WA Hubbard et al, Physical Review Applied, 10 (2018), p. 044066

[3] WA Hubbard et al., Applied Physics Letters 115 (2019), p. 133502. 
[4] M Mecklenburg et al, Ultramicroscopy, 207 (2019), p. 112852.

[5] M Mecklenburg et al., Science 347 (2015), p. 629-632.

[6] This material is based upon work supported by the Defense Microelectronic Activity under Contract No. HQ072720P0004, and by NSF STC award DMR-1548924 (STROBE), NSF award DMR-2004897, the UCLA PSEIF, and award 70NANB20H117 from NIST, U.S. Dept. of Commerce. 